OPEN ACCESS

Edited by:

Arifullah Mohammed, Universiti Malaysia Kelantan, Malaysia

Reviewed by:

Sirajudeen KNS,

International Islamic University

Malaysia, Malaysia

Mukhtar Ahmed,

King Saud University, Saudi Arabia

Yavuz Erden,

Bartin University, Turkey

*Correspondence:

Suresh V. Chinn

v_suresh@aimst.edu.my;

cvsureshgupta@gmail.com

tThese authors have contributed equally to this work

Specialty section:

This article was submitted to Integrative Physiology,

a section of the journal

Frontiers in Physiology

Received: 23 July 2021

Accepted: 31 August 2021

Published: 21 September 2021

Citation:

Sengupta P, Dutta S, Karkada IR, Akhigbe RE and Chinni SV (2021)

Irisin, Energy Homeostasis and Male

Reproduction

Front. Physiol. 12:746049.

doi: 10.3389/fphys.2021.746049

\section{Irisin, Energy Homeostasis and Male Reproduction}

\author{
Pallav Sengupta ${ }^{1 \dagger}$, Sulagna Dutta ${ }^{2 \dagger}$, Ivan Rolland Karkada ${ }^{1 \dagger}$, \\ Roland Eghoghosoa Akhigbe ${ }^{3,4}$ and Suresh V. Chinni5*
}

\begin{abstract}
${ }^{1}$ Physiology Unit, Faculty of Medicine, Bioscience and Nursing, MAHSA University, Kuala Lumpur, Malaysia, ${ }^{2}$ Department of Oral Biology and Biomedical Sciences, Faculty of Dentistry, MAHSA University, Kuala Lumpur, Malaysia, ${ }^{3}$ Department of Physiology, Ladoke Akintola University of Technology, Ogbomoso, Nigeria, ${ }^{4}$ Reproductive Biology and Toxicology Research Laboratories, Oasis of Grace Hospital, Osogbo, Nigeria, ${ }^{5}$ Department of Biotechnology, Faculty of Applied Sciences, AIMST University, Bedong, Malaysia
\end{abstract}

Irisin is a novel skeletal muscle- and adipose tissue-secreted peptide. It is conventionally regarded as an adipomyokine and is a cleaved fragment of Fibronectin type III domaincontaining protein 5 (FNDC5). It is involved in the browning of white adipose tissue, glucose tolerance, and reversing of metabolic disruptions. Fertility is closely linked to energy metabolism and the endocrine function of the adipose tissue. Moreover, there is established association between obesity and male infertility. Irisin bears strong therapeutic promise in obesity and its associated disorders, as well as shown to improve male reproductive functions. Thus, irisin is a molecule of great interest in exploring the amelioration of metabolic syndrome or obesity-induced male infertility. In this review we aim to enumerate the most significant aspects of irisin actions and discuss its involvement in energy homeostasis and male reproduction. Though current and future research on irisin is very promiscuous, a number of clarifications are still needed to reveal its full potential as a significant medicinal target in several human diseases including male infertility.

Keywords: energy metabolism, irisin, male infertility, reproduction, metabolic syndrome

\section{INTRODUCTION}

Metabolic syndrome and energy dyshomeostasis are among the major disruptors of male reproductive health (Morrison and Brannigan, 2015). Obesity links with male infertility by multitudinous mechanisms (Katib, 2015; Bhattacharya et al., 2020). Men with metabolic disorders often have disturbed levels of reproductive hormones, most prominently with low testosterone and high estradiol (Pauli et al., 2008). These hormonal imbalance reportedly corresponds to the severity of metabolic syndrome (Rosenblatt et al., 2015). Insulin Resistance (IR), inflammation and Oxidative Stress (OS) also are underlying players in the mechanism how excess body fat disrupts reproductive functions (Morrison and Brannigan, 2015).

Various classical and non-classical hormones and factors have been discussed in bridging the knowledge gap among metabolic disorders, energy dyshomeostasis and male infertility (Alahmar et al., 2019; Bhattacharya et al., 2019; Dutta et al., 2019a,b,c; İrez et al., 2019; Sengupta et al., 2019a,b). Irisin, discovered by Boström et al. (2012), is a novel myokine/adipokine secreted by skeletal muscle as well as adipose tissues. Irisin is a remarkable molecule which is mainly induced via exercise, and in the adipose tissues, it converts white adipocytes into metabolically 
active brown adipocytes, thereby holding promise as a therapeutic in obesity (Zhang et al., 2014, 2016b). Irisin has been shown to improve insulin sensitivity, enhancing cognitive capacities, thereby reversing metabolic imbalances and associated disorders (Perakakis et al., 2017). Despite its essential contribution in energy homeostasis, its detailed physiological actions on reproduction are yet to be explored. Few studies on animals indicate positive impact of irisin upon male fertility (Nanees and Reham, 2018; Tekin et al., 2019; Luo et al., 2021). Thus, it may be assumed that irisin, via reversing obesity, also ameliorates the obesity-induced disruptions in male fertility. The present article thus reviews the available literature pertaining to the versatile roles of irisin in metabolism, energy homeostasis and male reproduction and discusses the possible involved mechanisms.

\section{IRISIN: AN ADIPO-MYOKINE AND ITS RECEPTORS}

The precursor of irisin is a transmembrane glycoprotein, Fibronectin type III domain-containing protein 5 (FNDC5) which was detected for the first time in 2002 (Ferrer-Martínez et al., 2002; Teufel et al., 2002), and is also called FRCP2 and Pep. The FNDC5 proteolytic cleavage produces irisin in response to Peroxisome proliferator-activated receptor gamma coactivator 1 alpha (PGC-1 $\alpha$ ) activation (Boström et al., 2012). Irisin bears a molecular weight of $12 \mathrm{kDa}$ with 112 amino acid residues (Boström et al., 2012), while the structure of irisin is yet to fully revealed.

Irisin was discovered through a study in search of factors secreted in response to PGC- $1 \alpha$ by the skeletal muscles (Schumacher et al., 2013). PGC-1 $\alpha$ mediates the physiological benefits of exercise such as white-to-brown fat conversion (Handschin and Spiegelman, 2008), improvement of insulin sensitivity and signaling (Wenz et al., 2009). Studies have also demonstrated that irisin is primarily released in response to exercise (Boström et al., 2012; Huh et al., 2012).

Barely 6 years after the discovery of irisin, Kim et al. (2018) clearly demonstrated that the physiological actions of irisin are mediated via $\alpha \mathrm{V}$ integrins located in osteocytes, myocytes, and adipose tissues. They showed that irisin therapy ameliorated hydrogen peroxide-induced apoptosis in MLO-Y4 (osteocytelike) cell-line, demonstrating that irisin confers protection against apoptosis and induces bone resorption by upregulating sclerostin (Kim et al., 2018). In addition, they demonstrated that FNDC5knockout mice had significantly lower expression of receptor activator of nuclear factor kappa-B ligand (RANKL) mRNA (Kim et al., 2018). Quantitative proteomics analyses in MLOY4 osteocytes identified five cell surface proteins as possible receptor candidates for irisin. Among them, only integrin $\beta 1$ which binds with $\alpha$-integrins to form obligate heterodimers, is known to trigger downstream signaling; phosphorylation of focal adhesion kinase (FAK), protein kinase B (AKT), and cyclic AMP (cAMP) response element-binding protein (CREB) (Schaller et al., 1994; Giancotti and Ruoslahti, 1999; D’Amico et al., 2000). Irisin-treated MLO-Y4 cells showed phosphorylation of FAK, AKT, CREB, and Zyxin (Kim et al., 2018). This infers that irisin activates a pathway of integrin-like signaling. The $\alpha \mathrm{V} / \beta 5$ integrin had the highest binding affinity while other integrin complexes showed weak binding to irisin. Their quantitative proteomics with mass spectrometry revealed that $\alpha \mathrm{V}$ is the most abundant integrin protein in MLO-Y4 cells followed by integrin $\beta 1$, integrin $\alpha 5$, integrin $\beta 5$, integrin $\beta 3$, integrin $\beta 6$, and integrin $\beta 8$. Furthermore, it was reported that HEK293T cells with forced expression of integrin $\alpha \mathrm{V} / \beta 5$ but not $\alpha \mathrm{V} / \beta 3$ showed enhanced FAK phosphorylation with irisin treatment. Inhibition of $\alpha \mathrm{V} / \beta 5$ absolutely blocked the observed irisindriven phosphorylation of FAK, CREB, and Zyxin. This finding highlights the role of integrin $\alpha \mathrm{V} / \beta 5$ in irisin-mediated functions (Kim et al., 2018).

Although the physiological roles of irisin are still evolving, recent studies have shown that irisin secretion is not limited to the osteocyte, myocytes and adipose tissue as it has been found in a variety of tissues. In central nervous system (CNS), irisin expression has been detected in the Purkinje cells of the cerebellum (Dun et al., 2013), spinal cord and cerebral cortex (Huh et al., 2012). In peripheral tissues, it is reported in liver, kidney (Aydin, 2014), salivary glands (Aydin et al., 2013), cardiac muscles (Aydin et al., 2017), skin and testis (Aydin et al., 2014). Among the male reproductive tissues, testis bears the highest irisin expressions followed by prostate gland, while within testis, irisin is mostly expressed in the developing germ cells, peritubular cells and Leydig cells (The Human Protein Atlas, 2021; Figure 1).

\section{IRISIN IN ENERGY HOMEOSTASIS AND METABOLIC SYNDROME}

Irisin partially bridges the knowledge gap on the interactions of working tissues with other tissues to mediate energy homeostasis. Although irisin is primarily known as a myokine, it is also released from adipose tissue (Moreno-Navarrete et al., 2013; Roca-Rivada et al., 2013), earning its name as an adipokine. It mediates the beneficial metabolic effects of exercise (Grygiel-Górniak and Puszczewicz, 2017). Irisin induces the expression of mitochondrial uncoupling protein 1 (UCP1) and conversion of white to brown adipose tissue, resulting in raised energy expenditure by increased thermogenesis (Zhang et al., 2017). Thus, irisin holds promise to be a therapeutic molecule in mitigation of metabolic syndrome and related disorders.

Irisin aids skeletal muscle glucose uptake, facilitate glucose and lipid metabolism in liver, serving as insulin sensitizing hormone and reversing conditions of hyperlipidemia and hyperglycemia in metabolic disorders (Chen et al., 2016). Recent studies depicted that irisin stimulates glucose uptake in muscle cells via the calcium/ROS and P38/AMP activated protein kinase (AMPK) mediated pathway (Mu et al., 2001; Zhang et al., 2014; Figure 2). Irisin can ameliorate insulin resistance (IR) by its influence on the functions of the tissues, mainly liver and pancreas, that are involved in the etiology of type 2 diabetes (Moreno-Navarrete et al., 2013; Chen et al., 2016). Moreover, thyroid hormones play significant roles in metabolism, 

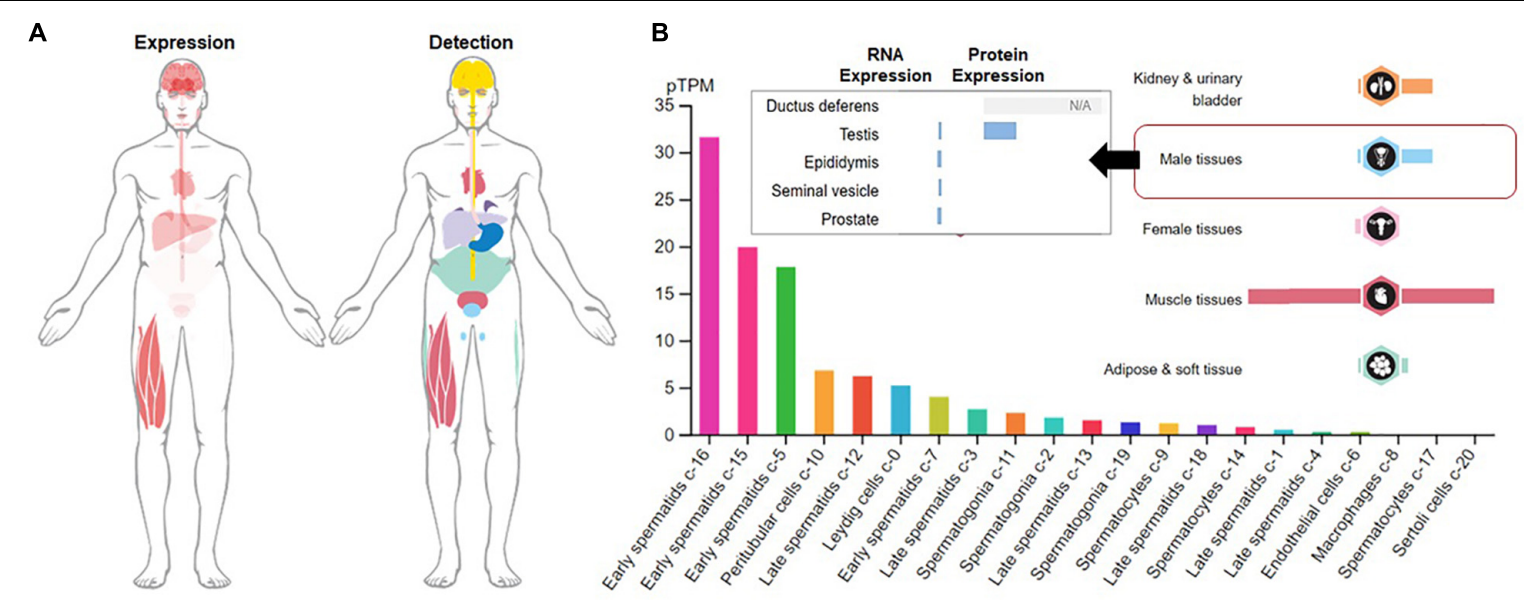

FIGURE 1 | Expression and detection of Fibronectin type III domain containing 5 (FNDC5). (A) Global expression and detection of FNDC5 RNA/protein; (B) FNDC5 RNA (normalized expression) and protein expression in overall male reproductive tissues and specific testicular cells.

and it is reported that triiodothyronine (T3) can increase the levels of adiponectin and leptin as well as improve insulin sensitivity, while irisin has least impact on adipokines levels, but it plays role in prevention of obesity or body weight regulation owing to its effects upon lipid profile (De Oliveira et al., 2020). Despite, the fact that central irisin administration inhibits the hypothalamic-pituitary-thyroid axis, it appears to be a key regulator of food intake and energy metabolism (Tekin et al., 2018). As irisin impacts on hypothalamic-pituitarythyroid axis, it indirectly may impact on reproductive functions (Sengupta and Dutta, 2018).

In mature adipocytes, irisin excites white-to-brown fat conversion by elevating UCP1 and PR/SET Domain 16 (PRDM16) via upregulation of $\mathrm{p} 38$ mitogen-activated protein kinase (MAPK) and extracellular signal-regulated kinase (ERK) signaling (Boström et al., 2012; Raschke et al., 2013; Huh et al., 2014; Zhang et al., 2014, 2016a). Several studies examined the link between circulating irisin, adiposity, and obesity in humans but with inconsistent results, which may be explained by the fact that irisin may be involved in compensatory mechanism for altered metabolism in obesity and thereby different metabolic status of the specific obese individual determines its levels. Some studies reported a positive correlation between serum irisin levels, body mass index (BMI) and adiposity (Stengel et al., 2013; Crujeiras et al., 2014b), whereas others found inverse association among the circulating irisin levels, BMI and the amount of fat tissue or could not detect any significant change in circulating irisin levels (Huh et al., 2012; Gouni-Berthold et al., 2013). Positive relation of irisin with fat mass, waist circumference, waistto-hip ratio and leptin levels have also been evidenced in obese subjects (Stengel et al., 2013; Crujeiras et al., 2014b) while a negative association was shown between irisin and adiponectin (Blüher et al., 2014). Furthermore, irisin levels were significantly reduced following weight loss due to bariatric surgery, an effect attributed to a lower fat-free mass and decreased FNDC5 mRNA expression in skeletal muscle (Huh et al., 2012). On the other hand, the reduction in irisin levels was reversed in patients who regained their original weight (Crujeiras et al., 2014a). This suggests that elevated irisin levels could be a compensatory mechanism for the abnormal metabolism and insulin sensitivity characteristic of obese individuals (Huh et al., 2012). Obesity is characterized by systemic inflammation (Bhattacharya et al., 2020), significant imbalance in cytokine secretion that is a strong predictor of developing IR and type-2 diabetes (Fantuzzi, 2005). In addition to cytokines, the activated toll-like receptor 4 (TLR4) is also strongly associated with IR as it increases TNF- $\alpha$ expression, that in turn affects insulin signaling pathway in muscle and adipose tissue (Könner and Brüning, 2011). Interestingly, irisin treatment suppressed expression of pro-inflammatory cytokines, nuclear factor-kappa B (NF- $\kappa \mathrm{B})$, TNF- $\alpha$, and IL-6 in a concentration dependent manner. Irisin reduced MCP1 expression in the cultured adipocytes which subsequently attenuated migration of macrophages in the presence of irisin. Moreover, irisin induced the phenotypic switching of adipose tissue macrophages from M1 (pro-inflammatory) to M2 (antiinflammatory) state (Dong et al., 2016). Therefore, FNDC5/irisin expression is associated with some anti-inflammatory markers (Moreno-Navarrete et al., 2013).

\section{IRISIN ENERGY HOMEOSTASIS AND MALE REPRODUCTIVE FUNCTIONS}

Report by Kim et al. (2018) upended most of the conflicting data on irisin receptor, but it also raises questions and opens up studies in other fields. Are $\alpha \mathrm{V} / \beta 5$ integrin receptors expressed in the male reproductive tract? If yes, what are their specific functions? Would inhibition of irisin (or FNDC5) or its binding to $\alpha \mathrm{V} / \beta 5$ affect male fertility adversely? (Kim et al., 2018). Albeit available data establishing a link between irisin and male reproductive function is scarce, its secretion in the seminal vesicle, penis, and testis (Huh et al., 2012; 


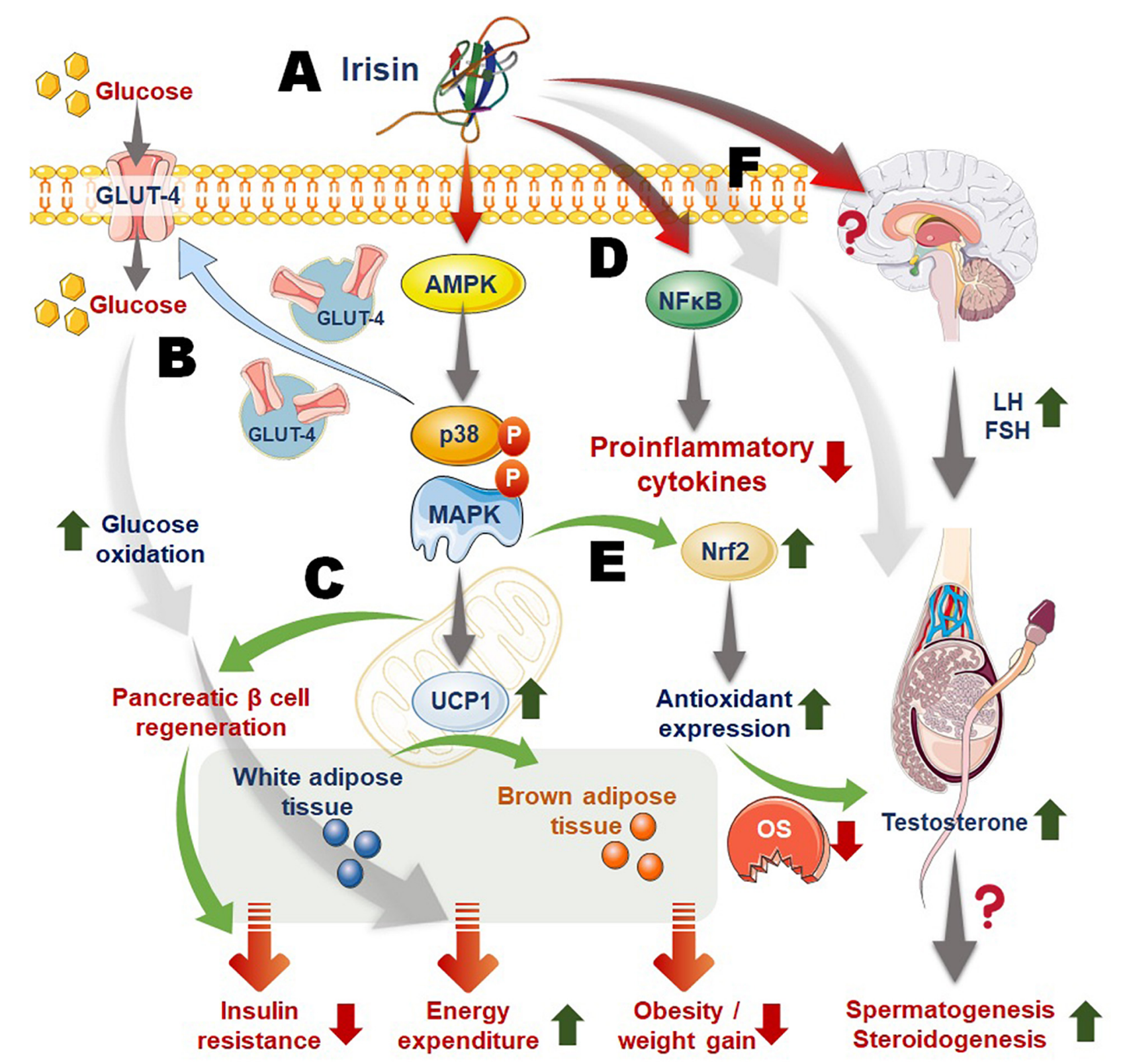

FIGURE 2 | Mechanism of irisin actions linking energy homeostasis, obesity, inflammation, and male reproduction. (A) Irisin acts via activation of the AMP activated protein kinase (AMPK), P38, MAPK (mitogen activated protein kinase) pathway; (B) irisin-activated pathway upregulates glucose transporter 4 (GLUT4) expression and transportation to membrane, aiding cellular glucose uptake that follows increased glucose metabolism and energy expenditure; (C) irisin also induces the expression of mitochondrial uncoupling protein 1 (UCP1) that aids conversion of white adipose tissue to brown adipose tissue, resulting in raised total body energy expenditure, as well as facilitates pancreatic $\beta$-cell regeneration that contribute to irisin-mediated reversing of insulin resistance; (D) irisin downregulates nuclear factor kappa-B (NF-kB) thereby playing role in suppressing inflammatory responses; (E) irisin-induced activation of Nrf2 (nuclear factor erythroid-2 related factor) may increase production of antioxidant enzymes thereby curbing excess reactive oxygen species (ROS) and oxidative stress (OS); (F) irisin may act on the HPG (hypothalamic-pituitary-gonadal) axis or directly upon the testicular cells to regulate male reproductive functions. Moreover, irisin actions to improve metabolic balance as well as to reverse obesity, inflammation and OS, may confer ameliorative impact upon obesity/inflammation/OS-mediated male infertility.

Aydin, 2014) might infer that it exerts some autocrine and paracrine effects on the male reproductive function. In addition, since energy balance has been established to play key roles in maintaining optimal reproductive function, irisin-driven energy homeostasis may be beneficial to the male reproductive function (Figure 2).

\section{IRISIN AND STEROIDOGENESIS}

The hypothalamic-pituitary-gonadal (HPG) axis is the main endocrine regulator of the male reproductive functions (Akhigbe et al., 2020). The hypothalamic signal to the pituitary gland is via the gonadotropin-releasing hormone (GnRH) 
(Dhillo et al., 2005; Corradi et al., 2016). The pituitary gland sends signal to the testis by releasing follicle stimulating hormone (FSH) and luteinizing hormone (LH) from the pituitary gonadotrophs. FSH and LH exert their effects on the testis by binding to FSH-R that is predominantly expressed in the Sertoli cells within the seminiferous tubules and LH-R that is expressed in the interstitial Leydig cells, respectively (Ramaswamy and Weinbauer, 2014). In response to $\mathrm{LH}$ signaling, the conversion of cholesterol into testosterone through series of biochemical events (Akhigbe et al., 2020). The gonadotropins establish the adult population of Sertoli, Leydig and stem germ cells and their functions, thus maintaining normal spermatogenesis (Ramaswamy and Weinbauer, 2014). Energy dyshomeostasis has been associated with upregulation of estrogen receptor expression in the male hypothalamus (Chimento et al., 2014). In turn, this triggers a negative feedback mechanism and inhibits the pulsatile release of $\mathrm{GnRH}$, resulting in decline in FSH and $\mathrm{LH}$ release and impaired testosterone production. Energy dyshomeostasis also increases the level of aromatase, which raises the conversion of testosterone to estrogen, thus inhibiting testicular function and suppressing circulatory androgen (Hammoud et al., 2006).

Irisin (and FNDC5), possibly via elevation of expression of mitochondrial UCP1, activates thermogenesis and lipolysis with resultant maintenance of energy balance. This might downregulate the expression of estrogen receptor in the hypothalamus, ensuring optimal pulsatile $\mathrm{GnRH}$ release and consequent $\mathrm{FSH}$ and $\mathrm{LH}$ release into the circulatory, resulting in Leydig cell-dependent testosterone production. Re-establishment of energy balance by irisin may also inhibit the conversion of testosterone to estrogen via repression of aromatase activity. Irisin-led elevation of UCP1 and PR/SET Domain 16 (PRDM16) via upregulation of p38 MAPK and ERK signaling (Boström et al., 2012; Huh et al., 2014; Zhang et al., 2014, 2016a) may not only cause white-to-brown fat conversion, but also blunt estrogen-induced cytokine-mediated inflammation.

It is possible that irisin-induced upregulation of p38 MAPK and ERK signaling activates nuclear factor erythroid-2 related factor (Nrf2) (Zhao et al., 2014), resulting in increased expression and activities of antioxidants and protection against ROS attack, oxidative stress and inflammation of the testis (Copple et al., 2017; Askari et al., 2018). Thus, it preserves testicular integrity and function, and promoting testosterone production by mitigating effects of oxidative stress (OS), which has been reported to induce inflammation, and vice versa (Akhigbe et al., 2020), with consequent apoptosis of the testicular tissue and testicular dysfunction.

Irisin may also exert regulatory role on the HPG axis. Kisspeptins, a family of peptides encoded by the KISS1 gene, has been reported to be expressed in the hypothalamus and testis among other tissues (West et al., 1998; Pinilla et al., 2012). The KISS1/GPR54 system plays a central role in the initiation of HPG axis, testosterone production, pubarche, and fertility maintenance (Navarro and Tena-Sempere, 2011). Kisspeptin system thus governs the HPG axis. Impaired expression of the kiss1 gene results in metabolic dysfunction and hypogonadism (Castellano et al., 2006). Reports suggest significant role of kisspeptin neuronal network in connecting energy homeostasis to the reproductive axis (Brown et al., 2008; Hameed et al., 2011). However, the exact mechanism of kisspeptin signaling is unclear. Moreover, kisspeptin signaling has also has a regulatory role in adipose tissue metabolism and it has been found to trigger irisin release (Shamas et al., 2019). Reports also showed that administration of irisin and kisspeptin increased neuropeptide Y (NPY) levels (Ferrante et al., 2016; Orlando et al., 2018) depicting the role of NPY in linking the kisspeptin and irisin pathways. Increase in irisin levels following kisspeptin administration also validate kisspeptin-mediated irisin release via direct irisin neurons stimulation in the hypothalamus or by the active skeletal muscles. It is also being suggested that the interactions between irisin and kisspeptin neurons are involved in regulation of reproductive functions (Tekin et al., 2019). The available reports suggest that irisin, when administered alone trigger reproductive hormones (Jiang et al., 2017), but mediate reverse effects when combined with other factors (such as $\mathrm{GnRH}$ and insulin) (Poretsky et al., 2017). Current studies are insufficient to elucidate the effects of irisin on the HPG axis and thus on reproductive functions. Current research is thus not enough to elucidate the impacts of irisin upon the HPG axis with reports claiming irisin to be inhibitory (Poretsky et al., 2017; Tekin et al., 2019), activator (Jiang et al., 2017), or ineffectual (Huh et al., 2014) on reproductive endocrine axis.

\section{IRISIN, SPERMATOGENESIS, AND SPERM QUALITY}

Energy dyshomeostasis-led androgen suppression adversely affects spermatogenesis via suppression of testosterone (Bieniek et al., 2016). This results in oligozoospermia and azoospermia (Sermondade et al., 2013). Irisin-mediated upregulation of the expression of Elov13, Cox7a, and Otop1 genes and increased energy expenditure maintain energy homeostasis (Boström et al., 2012). It has been shown that irisin administration in obese male rats could downregulate IR, decrease BMI, enhance the serum levels of FSH and $\mathrm{LH}$, increase testosterone levels thereby resulting in improved spermatogenesis and increased sperm parameters, namely sperm count and motility (Nanees and Reham, 2018). Moreover, in vitro study demonstrated the possible role of irisin in spermatogenesis owing to increased irisin expressions in Sertoli cells and undifferentiated spermatogonia transcripts in organotypic primate testicular tissue culture (Wahab et al., 2020).

Several studies have linked obesity with adverse male fertility profile (Jensen et al., 2004; Sermondade et al., 2013). The obesogenic environment stimulates various adipose tissuederived hormones, among which leptin is widely studied and rise in leptin following energy imbalance leads to increased circulatory estrogen levels, resulting in increased conversion of androgen to estrogen, thereby reducing testosterone levels (Fantuzzi, 2005; Sengupta et al., 2019a). It also reduces sex hormone-binding globulin production (Tsai et al., 2004) thereby restricting the availability of free testosterone. Obesity also mediates increase in pancreatic insulin production and peripheral tissue insulin resistance (Farooqi et al., 2003). Reports 
claim that obesity-induced altered testosterone production, spermatogenesis and semen quality may be carried out via some common mechanisms that involve OS (Sengupta et al., 2019a). The membranes of the sperm cells are rich in polyunsaturated fatty acid that predisposes them to reactive oxygen species (ROS) attack and oxidative damage including damaged sperm DNA integrity (Selvam et al., 2020). It is credible to suggest that irisin-induced activation of Nrf2 via upregulation of p38 MAPK and ERK signaling (Zhao et al., 2014) may confer protection against ROS attack and oxidative damage to the testis and the sperm cells, thereby enhancing spermatogenesis and sperm quality (Figure 2). Thus, the rise in energy consumption and thermogenesis along with the Nrf2 signaling induced by irisin (Zhang et al., 2014; Askari et al., 2018) would likely cause a decline in energy dyshomeostasis-driven rise in obesity-led oxidative damage. However, studies are needed to validate the most likely assumption that the above mentioned irisin signaling pathway may result in improved insulin sensitivity and sex hormonebinding globulin production, restore testosterone production and functions, spermatogenesis as well as semen quality.

\section{CONCLUSION}

Irisin is an important novel molecule to be investigated for regulation of metabolic syndrome-induced male infertility. This article describes the major elements of irisin functions and discusses the relevance of irisin in energy homeostasis and male

\section{REFERENCES}

Akhigbe, R. E., Ajayi, L. O., Adelakun, A. A., Olorunnisola, O. S., and Ajayi, A. F. (2020). Codeine-induced hepatic injury is via oxido-inflammatory damage and caspase-3-mediated apoptosis. Mol. Biol. Rep. 47, 9521-9530. doi: 10.1007/ s11033-020-05983-6

Alahmar, A., Dutta, S., and Sengupta, P. (2019). Thyroid hormones in male reproduction and infertility. Asian Pac. J. Reprod. 8, 203-210. doi: 10.4103/ 2305-0500.268135

Askari, H., Rajani, S. F., Poorebrahim, M., Haghi-Aminjan, H., Raeis-Abdollahi, E., and Abdollahi, M. (2018). A glance at the therapeutic potential of irisin against diseases involving inflammation, oxidative stress, and apoptosis: an introductory review. Pharmacol. Res. 129, 44-55. doi: 10.1016/j.phrs.2018.01. 012

Aydin, S. (2014). Three new players in energy regulation: preptin, adropin and irisin. Peptides 56, 94-110. doi: 10.1016/j.peptides.2014.03.021

Aydin, S., Aydin, S., Kuloglu, T., Yilmaz, M., Kalayci, M., Sahin, I., et al. (2013). Alterations of irisin concentrations in saliva and serum of obese and normalweight subjects, before and after $45 \mathrm{~min}$ of a Turkish bath or running. Peptides 50, 13-18. doi: 10.1016/j.peptides.2013.09.011

Aydin, S., Kuloglu, T., Aydin, S., Kalayci, M., Yilmaz, M., Cakmak, T., et al. (2014). A comprehensive immunohistochemical examination of the distribution of the fat-burning protein irisin in biological tissues. Peptides 61, 130-136. doi: 10.1016/j.peptides.2014.09.014

Aydin, S., Kuloglu, T., Aydin, S., Yardim, M., Azboy, D., Temizturk, Z., et al. (2017). The effect of iloprost and sildenafil, alone and in combination, on myocardial ischaemia and nitric oxide and irisin levels. Cardiovasc. J. Afr. 28, 389-396. doi: 10.5830/CVJA-2017-025

Bhattacharya, K., Sengupta, P., and Dutta, S. (2019). Role of melatonin in male reproduction. Asian Pac. J. Reprod. 8, 211-219. doi: 10.4103/2305-0500.26 8142

Bhattacharya, K., Sengupta, P., Dutta, S., and Karkada, I. R. (2020). Obesity, systemic inflammation and male infertility. Chem. Biol. Lett. 7, 92-98. reproduction. Irisin can reverse the adversities of metabolic syndrome-mediated disruptions of male fertility and ameliorates spermatogenesis and steroidogenesis, possibly via its direct and/or indirect beneficial impact of amending insulin resistance, inflammation, OS, imbalanced HPG axis and testicular functions. It thus may provide new approach to treat male reproductive disorders by addressing the root causes of infertility. In-depth investigations are needed to reveal the detailed irisin signaling pathways in regulation of male reproductive functions. While irisin holds high promise in bridging the knowledge gap between energy homeostasis and male fertility various facets await to be explored to show its full potential as a key molecule in reverting metabolic syndrome-induced male reproductive dyshomeostasis.

\section{AUTHOR CONTRIBUTIONS}

$\mathrm{SD}, \mathrm{PS}$, and IK contributed to design the review and conceived the study. SD, PS, IK, RA, and SC drafted, edited, and reviewed the manuscript. SC procured the grant for the publication. All the authors have given their consent for submission.

\section{FUNDING}

This work was supported by the FRGS, Malaysia, FRGS/1/2018/STG03/AIMST/02/1.

Bieniek, J. M., Kashanian, J. A., Deibert, C. M., Grober, E. D., Lo, K. C., Brannigan, R. E., et al. (2016). Influence of increasing body mass index on semen and reproductive hormonal parameters in a multi-institutional cohort of subfertile men. Fertil. Steril. 106, 1070-1075. doi: 10.1016/j.fertnstert.2016.06.041

Blüher, S., Panagiotou, G., Petroff, D., Markert, J., Wagner, A., Klemm, T., et al. (2014). Effects of a 1-year exercise and lifestyle intervention on irisin, adipokines, and inflammatory markers in obese children. Obesity 22, 17011708. doi: 10.1002/oby.20739

Boström, P., Wu, J., Jedrychowski, M. P., Korde, A., Ye, L., Lo, J. C., et al. (2012). A PGC1- $\alpha$-dependent myokine that drives brown-fat-like development of white fat and thermogenesis. Nature 481, 463-468. doi: 10.1038/nature10777

Brown, R., Imran, S., Ur, E., and Wilkinson, M. (2008). KiSS-1 mRNA in adipose tissue is regulated by sex hormones and food intake. Mol. Cell. Endocrinol. 281, 64-72. doi: 10.1016/j.mce.2007.10.011

Castellano, J. M., Navarro, V. M., Fernández-Fernández, R., Roa, J., Vigo, E., Pineda, R., et al. (2006). Expression of hypothalamic KiSS-1 system and rescue of defective gonadotropic responses by kisspeptin in streptozotocin-induced diabetic male rats. Diabetes 55, 2602-2610. doi: 10.2337/db05-1584

Chen, N., Li, Q., Liu, J., and Jia, S. (2016). Irisin, an exercise-induced myokine as a metabolic regulator: an updated narrative review. Diab. Metab. Res. Rev. 32, 51-59. doi: 10.1002/dmrr.2660

Chimento, A., Sirianni, R., Casaburi, I., and Pezzi, V. (2014). Role of estrogen receptors and g protein-coupled estrogen receptor in regulation of hypothalamus-pituitary-testis axis and spermatogenesis. Front. Endocrinol. 5:1. doi: 10.3389/fendo.2014.00001

Copple, I. M., Dinkova-Kostova, A. T., Kensler, T. W., Liby, K. T., and Wigley, W. C. (2017). NRF2 as an emerging therapeutic target. Oxid. Med. Cell. Longev. 2017:8165458. doi: 10.1155/2017/8165458

Corradi, P. F., Corradi, R. B., and Greene, L. W. (2016). Physiology of the hypothalamic pituitary gonadal axis in the male. Urol. Clin. North. Am. 43, 151-162. doi: 10.1016/j.ucl.2016.01.001

Crujeiras, A. B., Pardo, M., Arturo, R. R., Santiago, N. C., Zulet, M. A., Martínez, J. A., et al. (2014a). Longitudinal variation of circulating irisin after an energy 
restriction-induced weight loss and following weight regain in obese men and women. Am. J. Hum. Biol. 26, 198-207. doi: 10.1002/ajhb.22493

Crujeiras, A. B., Zulet, M. A., Lopez-Legarrea, P., De La Iglesia, R., Pardo, M., Carreira, M. C., et al. (2014b). Association between circulating irisin levels and the promotion of insulin resistance during the weight maintenance period after a dietary weight-lowering program in obese patients. Metabolism 63, 520-531. doi: 10.1016/j.metabol.2013.12.007

D’Amico, M., Hulit, J., Amanatullah, D. F., Zafonte, B. T., Albanese, C., Bouzahzah, B., et al. (2000). The integrin-linked kinase regulates the cyclin D1 gene through glycogen synthase kinase $3 \beta$ and cAMP-responsive element-binding protein-dependent pathways. J. Biol. Chem. 275, 32649-32657. doi: 10.1074/ jbc.M000643200

De Oliveira, M., Mathias, L. S., Rodrigues, B. M., Mariani, B. G., Graceli, J. B., De Sibio, M. T., et al. (2020). The roles of triiodothyronine and irisin in improving the lipid profile and directing the browning of human adipose subcutaneous cells. Mol. Cell. Endocrinol. 506:110744. doi: 10.1016/j.mce.2020.110744

Dhillo, W. S., Chaudhri, O. B., Patterson, M., Thompson, E. L., Murphy, K. G., Badman, M. K., et al. (2005). Kisspeptin-54 stimulates the hypothalamicpituitary gonadal axis in human males. J. Clin. Endocrinol. Metab. 90, 66096615. doi: 10.1210/jc.2005-1468

Dong, J., Dong, Y., Dong, Y., Chen, F., Mitch, W. E., and Zhang, L. (2016). Inhibition of myostatin in mice improves insulin sensitivity via irisin-mediated cross talk between muscle and adipose tissues. Int. J. Obes. 40, 434-442. doi: 10.1038/ijo.2015.200

Dun, S. L., Lyu, R. M., Chen, Y. H., Chang, J. K., Luo, J. J., and Dun, N. J. (2013). Irisin-immunoreactivity in neural and non-neural cells of the rodent. Neuroscience 240, 155-162. doi: 10.1016/j.neuroscience.2013.02.050

Dutta, S., Biswas, A., Sengupta, P., and Nwagha, U. (2019a). Ghrelin and male reproduction. Asian Pac. J. Reprod. 8, 227-232. doi: 10.4103/2305-0500.268144

Dutta, S., Sengupta, P., and Biswas, A. (2019b). Adiponectin in male reproduction and infertility. Asian Pac. J. Reprod. 8, 244-250. doi: 10.4103/2305-0500.268153

Dutta, S., Sengupta, P., and Muhamad, S. (2019c). Male reproductive hormones and semen quality. Asian Pac. J. Reprod. 8, 189-194. doi: 10.4103/2305-0500. 268132

Fantuzzi, G. (2005). Adipose tissue, adipokines, and inflammation. J. Allergy Clin. Immunol. 115, 911-919. doi: 10.1016/j.jaci.2005.02.023

Farooqi, I. S., Keogh, J. M., Yeo, G. S., Lank, E. J., Cheetham, T., and O'rahilly, S. (2003). Clinical spectrum of obesity and mutations in the melanocortin 4 receptor gene. N. Engl. J. Med. 348, 1085-1095. doi: 10.1056/NEJMoa022050

Ferrante, C., Orlando, G., Recinella, L., Leone, S., Chiavaroli, A., Di Nisio, C., et al. (2016). Central inhibitory effects on feeding induced by the adipo-myokine irisin. Eur. J. Pharmacol. 791, 389-394. doi: 10.1016/j.ejphar.2016.09.011

Ferrer-Martínez, A., Ruiz-Lozano, P., and Chien, K. R. (2002). Mouse PeP: a novel peroxisomal protein linked to myoblast differentiation and development. Dev. Dyn. 224, 154-167. doi: 10.1002/dvdy.10099

Giancotti, F. G., and Ruoslahti, E. (1999). Integrin signaling. Science 285, 1028 1033. doi: $10.1126 /$ science.285.5430.1028

Gouni-Berthold, I., Berthold, H. K., Huh, J. Y., Berman, R., Spenrath, N., Krone, W., et al. (2013). Effects of lipid-lowering drugs on irisin in human subjects in vivo and in human skeletal muscle cells ex vivo. PLoS One 8:e72858. doi: 10.1371/journal.pone.0072858

Grygiel-Górniak, B., and Puszczewicz, M. (2017). A review on irisin, a new protagonist that mediates muscle-adipose-bone-neuron connectivity. Eur. Rev. Med. Pharmacol. Sci. 21, 4687-4693.

Hameed, S., Jayasena, C. N., and Dhillo, W. S. (2011). Kisspeptin and fertility. J. Endocrinol. 208:97. doi: 10.1677/JOE-10-0265

Hammoud, A. O., Gibson, M., Peterson, C. M., Hamilton, B. D., and Carrell, D. T. (2006). Obesity and male reproductive potential. J. Androl. 27, 619-626. doi: 10.2164/jandrol.106.000125

Handschin, C., and Spiegelman, B. M. (2008). The role of exercise and PGC1 $\alpha$ in inflammation and chronic disease. Nature 454, 463-469. doi: 10.1038/ nature 07206

Huh, J., Dincer, F., Mesfum, E., and Mantzoros, C. (2014). Irisin stimulates muscle growth-related genes and regulates adipocyte differentiation and metabolism in humans. Int. J. Obes. 38, 1538-1544. doi: 10.1038/ijo.2014.42

Huh, J. Y., Panagiotou, G., Mougios, V., Brinkoetter, M., Vamvini, M. T., Schneider, B. E., et al. (2012). FNDC5 and irisin in humans: i. Predictors of circulating concentrations in serum and plasma and II. mRNA expression and circulating concentrations in response to weight loss and exercise. Metabolism. 61, 17251738. doi: 10.1016/j.metabol.2012.09.002

İrez, T., Karkada, I. R., Dutta, S., and Sengupta, P. (2019). Obestatin in male reproduction and infertility. Asian Pc. J. Reprod. 8, 239-243. doi: 10.4103/23050500.268146

Jensen, T. K., Andersson, A. M., Jørgensen, N., Andersen, A. G., Carlsen, E., Petersen, J. H., et al. (2004). Body mass index in relation to semen quality and reproductive hormones among 1,558 Danish men. Fertil. Steril. 82, 863-870. doi: 10.1016/j.fertnstert.2004.03.056

Jiang, Q., Zhang, Q., Lian, A., and Xu, Y. (2017). Irisin stimulates gonadotropins gene expression in tilapia (Oreochromis niloticus) pituitary cells. Anim. Reprod. Sci. 185, 140-147. doi: 10.1016/j.anireprosci.2017.06.018

Katib, A. (2015). Mechanisms linking obesity to male infertility. Centr. Eur. J. Urol. 68, 79-85. doi: 10.5173/ceju.2015.01.435

Kim, H., Wrann, C. D., Jedrychowski, M., Vidoni, S., Kitase, Y., Nagano, K., et al. (2018). Irisin mediates effects on bone and fat via $\alpha \mathrm{V}$ integrin receptors. Cell 175, 1756-1768. doi: 10.1016/j.cell.2018.10.025

Könner, A. C., and Brüning, J. C. (2011). Toll-like receptors: linking inflammation to metabolism. Trends Endocrinol. Metab. 22, 16-23. doi: 10.1016/j.tem.2010. 08.007

Luo, Y., Qiao, X., Ma, Y., Deng, H., Xu, C. C., and Xu, L. (2021). Irisin deletion induces a decrease in growth and fertility in mice. Reprod. Biol. Endocrinol. 19, 1-13. doi: 10.1186/s12958-021-00702-7

Moreno-Navarrete, J. M., Ortega, F., Serrano, M., Guerra, E., Pardo, G., Tinahones, F., et al. (2013). Irisin is expressed and produced by human muscle and adipose tissue in association with obesity and insulin resistance. J. Clin. Endocrinol. Metab. 98, E769-E778. doi: 10.1210/jc.2012-2749

Morrison, C. D., and Brannigan, R. E. (2015). Metabolic syndrome and infertility in men. Best Prac. Res. Clin. Obs. Gynaecol. 29, 507-515. doi: 10.1016/j.bpobgyn. 2014.10.006

Mu, J., Brozinick, J. T. Jr., Valladares, O., Bucan, M., and Birnbaum, M. J. (2001). A role for AMP-activated protein kinase in contraction- and hypoxia-regulated glucose transport in skeletal muscle. Mol. Cell. 7, 1085-1094. doi: 10.1016/ S1097-2765(01)00251-9

Nanees, F., and Reham, H. I. (2018). Role of irisin administration in modulating testicular function in adult obese albino rats. Med. J. Cairo Univ. 86, 4647-4655 doi: $10.21608 / \mathrm{mjcu} .2018 .65747$

Navarro, V. M., and Tena-Sempere, M. (2011). Neuroendocrine control by kisspeptins: role in metabolic regulation of fertility. Nat. Rev. Endocrinol. 8, 40-53. doi: 10.1038/nrendo.2011.147

Orlando, G., Leone, S., Ferrante, C., Chiavaroli, A., Mollica, A., Stefanucci, A., et al. (2018). Effects of kisspeptin-10 on hypothalamic neuropeptides and neurotransmitters involved in appetite control. Molecules 23:3071. doi: 10 . 3390/molecules23123071

Pauli, E. M., Legro, R. S., Demers, L. M., Kunselman, A. R., Dodson, W. C., and Lee, P. A. (2008). Diminished paternity and gonadal function with increasing obesity in men. Fertil. Steril. 90, 346-351. doi: 10.1016/j.fertnstert.2007. 06.046

Perakakis, N., Triantafyllou, G. A., Fernández-Real, J. M., Huh, J. Y., Park, K. H., Seufert, J., et al. (2017). Physiology and role of irisin in glucose homeostasis. Nat. Rev. Endocrinol. 13, 324-337. doi: 10.1038/nrendo.2016.221

Pinilla, L., Aguilar, E., Dieguez, C., Millar, R. P., and Tena-Sempere, M. (2012). Kisspeptins and reproduction: physiological roles and regulatory mechanisms. Physiol. Rev. 92, 1235-1316. doi: 10.1152/physrev.00037.2010

Poretsky, L., Islam, J., Avtanski, D., Lin, Y. K., Shen, Y.-L., Hirth, Y., et al. (2017). Reproductive effects of irisin: initial in vitro studies. Reprod. Biol. 17, 285-288. doi: 10.1016/j.repbio.2017.05.011

Ramaswamy, S., and Weinbauer, G. F. (2014). Endocrine control of spermatogenesis: Role of FSH and LH/ testosterone. Spermatogenesis 4:e996025. doi: 10.1080/21565562.2014.996025

Raschke, S., Elsen, M., Gassenhuber, H., Sommerfeld, M., Schwahn, U., Brockmann, B., et al. (2013). Evidence against a beneficial effect of irisin in humans. PloS One 8:e73680. doi: 10.1371/journal.pone.0073680

Roca-Rivada, A., Castelao, C., Senin, L. L., Landrove, M. O., Baltar, J., Crujeiras, A. B., et al. (2013). FNDC5/irisin is not only a myokine but also an adipokine. PloS One 8:e60563. doi: 10.1371/journal.pone.0060563

Rosenblatt, A., Faintuch, J., and Cecconello, I. (2015). Abnormalities of reproductive function in male obesity before and after bariatric surgery-a 
comprehensive review. Obes. Surg. 25, 1281-1292. doi: 10.1007/s11695-0151663-1

Schaller, M. D., Hildebrand, J. D., Shannon, J. D., Fox, J. W., Vines, R. R., and Parsons, J. T. (1994). Autophosphorylation of the focal adhesion kinase, pp125FAK, directs SH2-dependent binding of pp60src. Mol. Cell. Biol. 14, 1680-1688. doi: 10.1128/MCB.14.3.1680

Schumacher, M. A., Chinnam, N., Ohashi, T., Shah, R. S., and Erickson, H. P. (2013). The structure of irisin reveals a novel intersubunit $\beta$-sheet fibronectin type III (FNIII) dimer: implications for receptor activation. J. Biol. Chem. 288, 33738-33744. doi: 10.1074/jbc.M113.516641

Selvam, M. K. P., Sengupta, P., and Agarwal, A. (2020). Sperm DNA fragmentation and male infertility. Genetics of male infertility. New York, NY: Springer, 155-172. doi: 10.1007/978-3-030-37972-8_9

Sengupta, P., Bhattacharya, K., and Dutta, S. (2019a). Leptin and male reproduction. Asian Pac. J. Reprod. 8, 220-226. doi: 10.4103/2305-0500.268143

Sengupta, P., and Dutta, S. (2018). Thyroid disorders and semen quality. Biomed. Pharmacol. J. 11, 01-10. doi: 10.13005/bpj/1342

Sengupta, P., Dutta, S., Tusimin, M., and Karkada, I. R. (2019b). Orexins and male reproduction. Asian Pac. J. Reprod. 8, 233-238. doi: 10.4103/2305-0500.268145

Sermondade, N., Dupont, C., Faure, C., Boubaya, M., Cédrin-Durnerin, I., Chavatte-Palmer, P., et al. (2013). Body mass index is not associated with spermzona pellucida binding ability in subfertile males. Asian J. Androl. 15, 626-629. doi: 10.1038/aja.2013.10

Shamas, S., Rani, S., Afsheen, S., Shahab, M., Ejaz, R., Sadia, H., et al. (2019). Changes in irisin release in response to peripheral kisspeptin-10 administration in healthy and obese adult men. Acta Endocrinol. 15, 283-288. doi: 10.4183/ aeb.2019.283

Stengel, A., Hofmann, T., Goebel-Stengel, M., Elbelt, U., Kobelt, P., and Klapp, B. F. (2013). Circulating levels of irisin in patients with anorexia nervosa and different stages of obesity-correlation with body mass index. Peptides 39, 125-130. doi: 10.1016/j.peptides.2012.11.014

Tekin, S., Beytur, A., Erden, Y., Beytur, A., Cigremis, Y., Vardi, N., et al. (2019). Effects of intracerebroventricular administration of irisin on the hypothalamus-pituitary-gonadal axis in male rats. J. Cell. Physiol. 234, 88158824. doi: $10.1002 /$ jcp. 27541

Tekin, S., Erden, Y., Ozyalin, F., Onalan, E. E., Cigremis, Y., Colak, C., et al. (2018). Central irisin administration suppresses thyroid hormone production but increases energy consumption in rats. Neurosci. Lett. 674, 136-141. doi: 10.1016/j.neulet.2018.03.046

Teufel, A., Malik, N., Mukhopadhyay, M., and Westphal, H. (2002). Frcp1 and Frcp2, two novel fibronectin type III repeat containing genes. Gene 297, 79-83. doi: 10.1016/S0378-1119(02)00828-4

The Human Protein Atlas. (2021). FNDC5 (FRCP2). Available online at: https: //www.proteinatlas.org/ENSG00000160097-FNDC5 (accessed July 22, 2021).

Tsai, E. C., Matsumoto, A. M., Fujimoto, W. Y., and Boyko, E. J. (2004). Association of bioavailable, free, and total testosterone with insulin resistance: influence of sex hormone-binding globulin and body fat. Diab. Care 27, 861-868. doi: 10.2337/diacare.27.4.861
Wahab, F., Drummer, C., Mätz-Rensing, K., Fuchs, E., and Behr, R. (2020). Irisin is expressed by undifferentiated spermatogonia and modulates gene expression in organotypic primate testis cultures. Mol. Cell. Endocrinol. 504:110670. doi: 10.1016/j.mce.2019.110670

Wenz, T., Rossi, S. G., Rotundo, R. L., Spiegelman, B. M., and Moraes, C. T. (2009). Increased muscle PGC-1 $\alpha$ expression protects from sarcopenia and metabolic disease during aging. Proc. Natl. Acad. Sci. 106, 20405-20410. doi: 10.1073/pnas.0911570106

West, A., Vojta, P. J., Welch, D. R., and Weissman, B. E. (1998). Chromosome localization and genomic structure of the KiSS-1 metastasis suppressor gene (KISS1). Genomics 54, 145-148. doi: 10.1006/geno.1998.5566

Zhang, C., Ding, Z., Lv, G., Li, J., Zhou, P., and Zhang, J. (2016a). Lower irisin level in patients with type 2 diabetes mellitus: a case-control study and meta-analysis. J. Diab. 8, 56-62. doi: 10.1111/1753-0407.1 2256

Zhang, G., Sun, Q., and Liu, C. (2016b). Influencing factors of thermogenic adipose tissue activity. Front. Physiol. 7:29. doi: 10.3389/fphys.2016.0 0029

Zhang, J., Valverde, P., Zhu, X., Murray, D., Wu, Y., Yu, L., et al. (2017). Exerciseinduced irisin in bone and systemic irisin administration reveal new regulatory mechanisms of bone metabolism. Bone Res. 5, 1-14. doi: 10.1038/boneres.20 16.56

Zhang, Y., Li, R., Meng, Y., Li, S., Donelan, W., Zhao, Y., et al. (2014). Irisin stimulates browning of white adipocytes through mitogen-activated protein kinase p38 MAP kinase and ERK MAP kinase signaling. Diabetes 63, 514-525. doi: $10.2337 / \mathrm{db} 13-1106$

Zhao, Y., Li, J., Tang, Q., Zhang, P., Jing, L., Chen, C., et al. (2014). Regulation of extracellular signal-regulated kinase $1 / 2$ influences hippocampal neuronal survival in a rat model of diabetic cerebral ischemia. Neural Regen. Res. 9, 749-756. doi: 10.4103/1673-5374.131581

Conflict of Interest: The authors declare that the research was conducted in the absence of any commercial or financial relationships that could be construed as a potential conflict of interest.

Publisher's Note: All claims expressed in this article are solely those of the authors and do not necessarily represent those of their affiliated organizations, or those of the publisher, the editors and the reviewers. Any product that may be evaluated in this article, or claim that may be made by its manufacturer, is not guaranteed or endorsed by the publisher.

Copyright (c) 2021 Sengupta, Dutta, Karkada, Akhigbe and Chinni. This is an openaccess article distributed under the terms of the Creative Commons Attribution License (CC BY). The use, distribution or reproduction in other forums is permitted, provided the original author(s) and the copyright owner(s) are credited and that the original publication in this journal is cited, in accordance with accepted academic practice. No use, distribution or reproduction is permitted which does not comply with these terms. 